\title{
DUALITY THEORY FOR LOCALLY COMPACT GROUPS WTH PRECOMPACT CONJUGACY CLASSES. I: THE CHARACTER SPACE(1)
}

\author{
BY
}

TERJE SUND

\begin{abstract}
Let $G$ be a locally compact group, and let $\mathcal{X}(G)$ consist of the nonzero extreme points of the set of continuous, $G$-invariant, positive definite functions $f$ on $G$ such that $f(e) \leq 1 . x(G)$ is called the character space, and is given the topology of uniform convergence on compacta. The purpose of the present paper is to extend the main results from the duality theory of abelian groups and $[Z]$ groups to the class of $[F C]^{-}$groups (i.e., groups with precompact conjugacy classes), letting $X(G)$ play the role of the character group in the abelian theory. Some of our theorems are only proved for the class $[F D]^{-}\left(C[F C]^{-}\right)$. If $G \in[F C]^{-}$ then $\mathcal{X}(G) \approx \mathcal{X}(H)$ where $H$ is a certain $[F I A]^{-}$quotient group. Hence there is no loss of generality to study character spaces of $[F I A]^{-}$groups.
\end{abstract}

1. Introduction. The main purpose of this paper to is extend certain results from the duality theory of locally compact abelian groups and $[Z]$ groups to $[F C]^{-}$groups (i.e., groups possessing precompact conjugacy classes) [10], [24].

For $[F C]$ groups there are at least two natural structure spaces; the character space $\mathcal{X}(G)$ and the dual space $\hat{G}$ which may replace the dual group in the abelian theory. In this paper we shall concentrate on the space $X(G)$, whereas a similar discussion of $\hat{G}$ will appear in the 2 nd paper of this series.

Our investigations will depend on earlier work of Grosser, Mosak, and Moskowitz [10], Grosser and Moskowitz [11], Kaniuth [13], Kaniuth and Schlichting [14], Liukkonen [18], and Mosak [23].

Received by the editors February 5, 1974 and, in revised form, August 20, 1974, 22D10.

AMS (MOS) subject classifications (1970). Primary 22D35; Secondary 43A40,

Key words and phrases. $[F C]_{\mathbb{B}}^{-}$group, $[F I A]_{\mathbb{B}}^{-}$group, $(L)_{\mathbb{B}}$ group, $\mathbf{B}$ character, group extension.

(1) This paper constitutes part of the author's doctoral dissertation at the University of Minnesota, Minneapolis written under Professor S. Grosser. 
For convenience we list some fundamental notation and properties of the classes of groups which we are dealing with. Let $G$ be a locally compact topological group, not necessarily separable, and suppose $\mathcal{B}$ is a subgroup of the topologized automorphism group $\mathscr{Q}(G)$. We shall always assume that $\mathfrak{B}$ contains the group of inner automorphisms $g(G)$. The following, by now well-established, notation will be used to denote classes of locally compact groups.

$[I N]_{B}-G$ has a compact $\mathscr{B}$ invariant neighborhood of $e$.

$[S I N]_{\mathscr{B}}-G$ has a fundamental system of $\mathfrak{B}$ invariant neighborhoods of $e_{\text {. }}$

$[F I A]_{\bar{B}}$ - The automorphism group $\mathfrak{B}$ has compact closure in $\mathcal{Q}(G)$. One can prove that $[F I A]_{\mathcal{B}}^{-}=[S I N]_{B} \cap[F C]_{B}^{-}[11]$.

$[F C]_{\bar{B}}-G$ has precompact $B$ conjugacy classes.

$[F D]_{\mathscr{B}}-G$ has precompact $B$ commutator group $G^{\prime}$.

$[Z]_{B}$-Locally compact groups $G$ such that $G / Z^{\mathbb{B}}(G)$ is compact, where $Z^{\mathcal{B}}(G)$ is the $B$ center of $G$.

[MOORE]-G has only finite dimensional continuous unitary irreducible representations.

We refer to Grosser and Moskowitz [11] and Robertson [25] for relationship between the various classes of groups. One has $[F I A]_{\mathbb{B}}^{-} \subset[F C]_{\mathbb{B}}^{-} \mathrm{C}$ $[I N]_{B} .\left({ }^{2}\right)$ The first inclusion is clear from the above, and the last inclusion follows from Robertson's structure theorem for $[F C]_{\mathbf{B}}^{-}$groups (Liukkonen [18]), which says that $G$ is an $[F C]_{\Phi}^{-}$group if and only if $G$ satisfies an exact sequence of topological groups $(e) \rightarrow K \rightarrow G \rightarrow \mathbf{R}^{n} \oplus$ $D \rightarrow(e)$, where $K$ is compact and $B$ invariant and $D$ is a discrete $[F C]_{\mathscr{B}}$ group ( 3 ) and $\mathbf{R}^{n} \oplus D$ has precompact orbits under the group $B^{\prime}$ of induced actions of $\mathfrak{B}$. For our purposes a structure theorem due to Grosser and Moskowitz [11, Theorem (3.16)] will also be useful, namely $G$ is an $[F C]^{-}$ group implies $G$ satisfies an exact sequence of topological groups $(e) \rightarrow$ $P \rightarrow G \rightarrow \mathrm{R}^{n} \oplus A \rightarrow(e)$, where $P$ is the closed periodic $B$ invariant subgroup of $G$ ( $x \in G$ is periodic iff $x$ is contained in a compact subgroup of $G$. The periodic subgroup $P$ consists exactly of the periodic elements in $G$ ), and $A$ is discrete, abelian, and torsion free. We note that a locally compact group which satisfies such a sequence need not be an $[F C]_{\bar{B}}^{-}$group; let e.g. $G$ be a semidirect product of $Z_{2}^{\infty}$ (the weak direct product of countably

(2) One also has $[Z] \subset[F I A]^{-}$(for $B=g(G)$ ).

(3) We shall omit the bar - whenever the groups are discrete. 
many copies of $Z_{2}$ ) with the integers $Z$, where $Z$ acts on $\left(a_{n}\right)_{n} \in Z_{2}^{\infty}$ by shifting of coordinates: $\eta(k)\left(a_{n}\right)_{n}=\left(a_{n+k}\right)_{n}$, for $k \in \mathbf{Z}$. If $a_{n}=1$ for $n \neq 0$ and $a_{0}=-1$ then $\eta(Z)\left(a_{n}\right)_{n}$ equals $\left\{\left(a_{n}\right)_{n}: a_{n}=1\right.$ except for exactly one $n \in \mathbf{Z}\}$. Hence $\eta(Z)\left(a_{n}\right)_{n}$ is not precompact in $G$ and $G=Z_{2}^{\infty} \times{ }_{\eta} Z$ is not an $[F C]_{\bar{B}}$ group $(\Re=g(G)$ ).

If $P$ is a periodic $[F C]_{\bar{B}}^{-}$group, $P$ has a compact $B$ invariant open subgroup $K$ such that $P$ is an extension $(e) \rightarrow K \rightarrow P \rightarrow H \rightarrow(e)$, where $H$ is a discrete torsion group in $[F C]_{B}[18]$.

We turn now to the $\mathfrak{B}$ characters. Suppose $G$ is a unimodular locally compact group, and let $X^{\mathscr{B}}(G)$ denote the set of nonzero extreme points of the convex set of continuous positive definite $B$ invariant functions $\alpha$ on $G$, such that $\alpha(e) \leq 1$ ( $\alpha$ is $B$ invariant if $\alpha(\beta(g))=\alpha(g)$, all $\beta \in \mathfrak{B}, g \in G$ ). There is a bijective correspondence between $X_{(G)}=X^{S(G)}(G)$ and the set of all classes of quasi-equivalent factor representations of finite type of $G$; see Dixmier [5, 17.3.4]. We give $X^{\mathscr{B}}(G)$ the topology of uniform convergence on compacta in $G$. For $B=\mathscr{Y}(G)$ and $G \in[F I A]^{-}, X(G)$ is locally compact in this topology [13].

Before starting our investigations we give a summary of our results. Note that most of our duality theory in $\$ \$ 2$ and 3 was proved for $[Z]$ groups in [10] (if $G \in[Z]$ then $\hat{G}$ and $X(G)$ are isomorphic).( $\left.{ }^{4}\right)$ If nothing else is said $G$ will denote an $[F C]_{\mathfrak{B}}^{-}$group.

\section{TABLE OF CONTENTS.}

1. Introduction. (pp. 185-188)

2. The $\mathfrak{B}$ character space $\mathfrak{X}^{\mathscr{B}}(G)$. (pp. 188-197)

2.3. The connected component of $\beta \in X^{\mathscr{B}}(G)$ is $\mathcal{U}_{\beta, P}=\left\{\alpha \in \mathscr{X}^{\mathscr{B}}(G)\right.$ : $\left.\alpha_{p}=\beta_{p}\right\}$.

2.5. $X(G)$ is discrete iff $G$ is compact.

2.6. If $\hat{G}$ is compact then $G$ is discrete $\left(G \in[F I A]^{-}\right)$.

2.7. $\hat{G}$ is discrete iff $G$ is compact.

2.8. $X(G)$ is $\sigma$-compact if $G$ is a first countable $[F D]^{-}$group. If $\Upsilon(G)$ is $\sigma$-compact and $G \in[F I A]^{-}$then $G$ is 1 st countable.

2.10. Let $G \in[F I A]^{-}$. $X(G)$ has finitely many connected components iff the periodic subgroup $P$ is finite.

2.12. Let $G \in[F I A]^{-}, G$ is aperiodic iff $X(G)$ is connected.

2.13. $X(G)$ is totally disconnected iff $G$ is periodic.

${ }^{4}$ ) Our proofs use the theory of $[F C]^{-}$groups and they are different from the ones given for $[Z]$ groups in [10]. 
3. Further results on $\mathcal{X}(G)$. (pp. 197-201)

3.1. If $N$ is a compact normal subgroup of $G$ such that $G / N$ is abelian and first countable then the map $\lambda \rightarrow \beta \cdot \lambda ; X(G / N) \rightarrow \beta \cdot X(G / N)$ is an open and continuous group homomorphism.

3.2. Let $G \in[F D]^{-}$and let $G / \bar{G}^{\prime}$ be first countable. Then $G$ is $\sigma$ compact iff $X(G)$ is first countable.

3.3. $X(G)$ is locally Euclidean if $G$ is compactly generated. If $G \in[F D]^{-}$and $X(G)$ is locally Euclidean then $G$ is compactly generated.

3.4. $X^{\mathscr{B}}(G)$ is locally connected if $G$ possesses property $(L)_{\mathbb{B}}$.

3.5. Let $G \in[F D]^{-}$and suppose $\Upsilon(G)$ is locally connected. Then $G$ is an $(L)$ group.

I would like to thank my advisor Professor S. Grosser for his encouragement and our many stimulating discussions during the time in which this work was done. I am also indebted to Professor R. Mosak who read through the manuscript and pointed out some gaps.

After this paper had been submitted to the Transactions of the American Mathematical Society, we learned about results of D. Steiner that coincide, essentially, with the present ones (D. Steiner, Zur harmonischen Analyse klassenkompakter Gruppen, $\$ 6$, Doctoral dissertation, Munich Technical University, 1973, unpublished). Moreover, E. Kaniuth recently obtained stronger results for [SIN] groups (Topology in duals of SIN-groups, Math. Z. 134 (1973), 67-80).

2. The $B$ character space $X^{\mathscr{B}}(G)$. In this section we will study the topological properties of the character space $X^{\mathscr{B}}(G)$ for $[F C]_{\mathbb{B}}^{-}$groups, with emphasis on connectivity properties. As in the abelian case the periodic subgroup plays a key role. After characterizing the connected components of $X^{B}(G)$, Proposition (2.3), we derive as a consequence several duality results analogous to those for abelian groups and [Z] groups; see [24] and [10].

The first lemma is useful when one wants to prove results concerning the $\mathfrak{B}$ characters of $[F C]_{\mathscr{B}}^{-}$groups which already are known to hold for $[F I A]_{\mathscr{B}}^{-}$groups [19]. Let $G$ be an $[F C]_{\mathscr{B}}^{-}$group (where $B \supset g(G)$ ) and let $C$ denote the intersection of all compact $B$ invariant neighborhoods of $e$ in $G$. Then $C$ is a compact $B$ invariant subgroup of $G$ and $H=G / C$ is an $[F I A]_{\mathscr{B}}^{\prime}$ group where $\mathfrak{B}^{\prime}$ is the automorphism group of $H$ induced by $\mathfrak{B}$. Finally, let $\theta$ denote the canonical map of $G$ onto $G / C$. (See $[11$, Theorem (2.5)], for a proof of the fact that $C$ is a group.) 
(2.1). Lemma. With the above notation, the map $a \rightarrow \alpha \circ \theta$ is a homeomorphism of $X^{\mathbb{B}^{\prime}}(H)$ onto $X^{\mathbb{B}}(G)$.

Proof. This is part of Proposition 1.2 in [19].

If $G$ is as above and $\alpha \in \mathcal{X}^{\mathcal{B}}(G)$ we let $\mathcal{U}_{\alpha, K}^{B}=\left\{\beta \in \mathcal{X}^{\mathcal{B}}(G): \alpha_{K}=\beta_{K}\right\}$, for any subset $K$ of $G$. Here, as always, $\beta_{k}$ denotes the restriction of $\beta$ to $K$.

(2.2). Lemma. Let $G$ be an $[F C]_{\mathscr{B}}^{-}$group, where $\mathfrak{B} g(G)$, and let $K$ be any compact $B$ invariant subgroup of $G$ containing $C$, where $C$ denotes the intersection of all $\mathfrak{B}$ invariant neighborboods of $e$ in $G$. If a $\in \Upsilon^{\mathscr{B}}(G)$ then the set $\mathcal{U}_{a, K}^{\mathbb{B}}$ is open and closed in $\mathfrak{X}^{\mathfrak{B}}(G)$.

Proof. We will first prove the result with $\mathfrak{B}=\mathscr{g}(G)$. (In this case it will be unnecessary to assume $K \supset C_{\text {.) }}$ ) If $\beta \in X(G)$ we have $\beta_{K}=\int_{\mathscr{X}(K)} \sigma d \mu_{\beta}(\sigma)$ for a unique positive regular Borel measure $\mu_{\beta}$ on $X(K)$, since $\beta$ is positive definite and $K$ invariant [13, Satz 2]. Now $X(K)$ is discrete so $\beta_{K}=$ $\Sigma_{m}(\sigma) \sigma$, where $m(\sigma)$ is some nonnegative real number and the sum is taken over $\mathfrak{X}(K)$. We claim that $\lambda=\beta_{K} \in X^{\mathcal{C}}(K)$ where $\mathcal{C}=\mathscr{I}(G)_{K^{\circ}}{ }^{(5)}$

Let $r$ be a $G$ invariant positive definite continuous function on $K$ with $\tau(e) \leq 1$ and suppose $\tau \ll \lambda$ (i.e. $\lambda-\tau$ is positive definite). We shall prove that $r=k \lambda$ for some nonnegative number $k, 0 \leq k \leq 1$. Let now $\pi_{\lambda}$ and $\pi_{\tau}$ be nondegenerate cyclic unitary representations associated with $\lambda$ and $r$ respectively, and let $\operatorname{ker} \pi_{\lambda}$ and $k e r \pi_{r}$ be their kernels when considered as representations of the measure algebra $M(K)$. Since $r \ll \lambda$ we shall see that $\operatorname{ker} \pi_{\lambda} \subset \operatorname{ker} \pi_{\tau}$. Let therefore $\nu \in \operatorname{ker} \pi_{\lambda}$. Since $\operatorname{ker} \pi_{\lambda}$ is an ideal in $M(K)$ we have $\nu * f \in \operatorname{ker} \pi_{\lambda}$, all $f \in C(K)$. Hence, by definition of $\pi_{\lambda}$ and $\pi_{\tau}$, we have

$$
0=\lambda\left((\nu * f)^{*} *(\nu * f)\right) \geq r\left((\nu * f)^{*} *(\nu * f)\right) \geq 0, \text { all } f \in C(K),
$$

and this implies $\nu \in \operatorname{ker} \pi_{\tau}$, so that $\operatorname{ker} \pi_{\lambda} \subseteq \operatorname{ker} \pi_{\tau^{*}}$.

Recall now that $\beta \in \mathfrak{X}(G)$ and hence satisfies the character formula

$$
\int_{G} \beta\left(y^{-1} x\right) d \mu(y)=\beta(x) \int_{G} \beta\left(y^{-1}\right) d \mu(y)
$$

for all $x \in G$ and every central measure $\mu \in M(G)$ (Leptin [17, Satz 2]). In particular

(5) This assertion has been established elsewhere; see Kaniuth [13, proof of Satz 4]. We include a proof for completeness. 


$$
\int_{K} \lambda\left(y^{-1} k\right) d \mu(y)=\lambda(k) \int_{K} \lambda\left(y^{-1}\right) d \mu(y), \quad \text { all } k \in K \text {, }
$$

where $\mu$ is a central measure in $M(G)$ with support contained in $K$. Let $a_{\mu}=\int_{K} \lambda\left(y^{-1}\right) d \mu(y)$ and let $\delta_{e}$ denote the point mass at $e$. Then( $\left(^{6}\right)$

$$
\int_{K} \lambda\left(y^{-1}\right) d\left(\mu-a_{\mu} \delta_{e}\right)(y)=\int_{K} \lambda\left(y^{-1}\right) d \mu(y)-a_{\mu} \lambda(e)=0
$$

Hence( $\left.{ }^{7}\right) \mu-a_{\mu} \delta_{e} \in \operatorname{ker} \pi_{\lambda} \subset \operatorname{ker} \pi_{\tau^{\circ}}$. Since $\operatorname{ker} \pi_{\tau}$ is an ideal in $M(K)$ it follows that, for all $k \in K, \delta_{k^{-1}} *\left(\mu-a_{\mu} \delta_{e}\right) \in \operatorname{ker} \pi_{\tau}$, where $\delta_{k} * \nu(E)=$ $\nu\left(k^{-1} E\right)$ for any Borel set $E$ in $G$ and $\nu \in M(G)$. Thus

$$
0=\int_{K} \tau\left(y^{-1}\right) d\left(\mu-a_{\mu} \delta e^{)(k y)}=\int_{K} \tau\left(y^{-1} k\right) d \mu(y)-a_{\mu} \tau(k) .\right.
$$

If $C_{k}=\left\{\iota_{x}(k): \iota_{x} \in G(G)\right\}$ is the conjugacy-class of $k \in K$ there is an $g(G)$ invariant measure $\mu_{k}=\mu$ with support in $C_{k}^{-}$, see e.g. Leptin $[17,(7)]$. Since $\lambda$ and $\tau$ are constant on $C_{k}^{-}$, it follows from (1) that (we normalize the measure)

$$
a_{\mu}=a_{\mu} \lambda(e)=\int_{C_{\bar{k}}^{-}} \lambda\left(y^{-1}\right) d \mu(y)=\lambda\left(k^{-1}\right),
$$

and, analogously, (from (1'))

$$
a_{\mu} \tau(e)=\int_{C_{\vec{k}}} \tau\left(y^{-1}\right) d \mu(y)=\tau\left(k^{-1}\right) .
$$

Hence $\tau(k)=\lambda(k) \tau(e)$ for all $k \in K$, and $\lambda$ must be an extreme point [23, 4.1], $[5,2.5]$, i.e., $\lambda \in \mathfrak{X}(K)$. Recall that $\lambda=\beta_{K}=\Sigma m(\sigma) \sigma$, and let $\sigma \in \mathcal{X}(K)$ be such that $m(\sigma) \neq 0$. By [23, Theorem 5.8] $\lambda=\sigma^{\#}=\int_{\bar{e}} \sigma \circ a d \alpha$, and since $\mathscr{X}(K)$ is discrete this integral reduces to a sum over the $\overline{\mathcal{C}}$ orbit $\theta$ of $\sigma$ : $\beta_{K}=1 / n \Sigma_{r, \tau} \in \theta$, where $n=m(\sigma)^{-1}$ is the finite order of $\theta$.

If now $a \in X_{(G)}$ and $\alpha_{K} \neq \beta_{K}$, then $\alpha_{K}$ must be concentrated on a different orbit $\theta_{1}$ in $X_{(K)}$; that is, $\theta_{1} \cap \theta=\varnothing$. Hence it follows from the orthogonality relations for characters that $\left({ }^{8}\right)$

(6) The following argument is due to Leptin [17].

( 7$)$ The measure $\nu=\mu-a_{\mu} \delta_{e}$ is central. Hence, for $x \in G,\langle\pi(\nu) \pi(x) u$, $\pi(x) u\rangle=\left\langle\pi(x)^{-1} \pi(x) \pi(\nu) u, u\right\rangle=\langle\pi(\nu) u, u\rangle=0$, and since $u$ is a cyclic vector for $\pi$ we have $\nu \in \operatorname{ker} \pi$ (where $\pi=\pi_{\lambda}$ ).

(8) The arguments used on the next page are certainly well known; see e.g. $[14$, p. 340 , the last paragraph]. 


$$
\int_{K} a(k) \beta(k)^{-} d k=0 .
$$

We next show that if $\alpha, \beta \in \Upsilon(G)$ and

$$
|\beta(k)-\alpha(k)|<\left[\int_{K}|\alpha(k)|^{2} d k\right]^{1 / 2}, \quad k \in K,
$$

then $\beta_{K}=a_{K}$. From this it will follow that $\mathcal{U}_{\alpha, K}$ is open in $X_{(G) \text {. Since }}$ $X(G)=\bigcup_{a} \mathcal{U}_{a, K}$ (disjoint union), each $\mathcal{U}_{a, K}$ must also be closed. If (3) holds we have

$$
\int_{K}|\beta(k)-\alpha(k)|^{2} d k<\int_{K}\left(\int_{K}|\alpha(k)|^{2} d k\right) d k=\int_{K}|\alpha(k)|^{2} d k
$$

(We use normalized Haar measure $d k$ on $K_{0}$ ) Now, if $\beta_{K} \neq a_{K}$, (2) gives

$$
\int_{K}|\beta(k)-\alpha(k)|^{2} d k=\int_{K}|\beta(k)|^{2} d k+\int_{K}|\alpha(k)|^{2} d k>\int_{K}|\alpha(k)|^{2} d k .
$$

Hence $\alpha_{K}=\beta_{K}$, and the proof is complete for the case $\mathfrak{B}=\mathscr{I}(G)$.

Suppose $B \supset g(G)$. With the notation as in (2.1), we lẹt $H$ be the $[F I A]_{\mathcal{B}^{\prime}}$ quotient group of $G$. Theorem (5.8) of Mosak [23] yields an open and continuous surjection $w: X(H) \rightarrow X^{\mathcal{B}^{\prime}}(H), \phi \rightarrow \int_{\overline{\mathbb{B}}}$, $\dot{\phi} \iota d \iota$, where $d \iota$ is normalized Haar measure on the compact automorphism group $\bar{\Phi}^{\prime}$. Letting $K^{\prime}=K / C$ and $\alpha \in X_{(H)}$ we see that $\left\{\beta \in \mathscr{X}(H): \beta_{K^{\prime}}=\alpha_{K^{\prime}}\right\}=\mathcal{U}_{\alpha, K^{\prime}}$ is open and closed by the above. Moreover, it is easy to see that $w$ maps $\mathcal{U}_{a, K^{\prime}}$ in $\mathscr{X}_{(H) \text { onto }}$

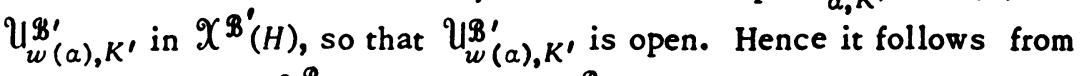
Lemma (2.1) that $\mathcal{U}_{\alpha^{\prime}, K}^{\mathscr{B}}$ is open in $\Upsilon^{\mathscr{B}}(G)$, where $\alpha^{\prime}$ denotes the image of $w(a)$ under the canonical homeomorphism $\mathfrak{X}^{B^{\prime}}(H) \rightarrow \Upsilon^{\mathbb{B}}(G)$. Since $\mathfrak{X}^{\mathbb{B}}(G)$ is the disjoint union of the different $\mathcal{U}_{a^{\prime}, K}^{\mathbb{B}}$, it follows that each $\mathcal{U}_{a^{\prime}, K}^{\mathbb{B}}$ is also closed, and the proof is complete.

We are now in a position to characterize the connected components of $X^{\mathscr{B}}(G)$ when $G$ is an $[F C]_{\mathscr{B}}^{-}$group. Our result is analogous to the one for $[Z]$ groups (see $[10,2.2]$ ).

Let $P$ be the periodic subgroup of $G$. Since the intersection $C$ of all $B$ invariant neighborhoods of $e$ in $G$ is compact, one has $C \subset P$ and $G / P$ is an $[F I A]_{\mathscr{B}^{\prime}}$ group. Hence the canonical map $X(G / P) \rightarrow \Upsilon^{\mathscr{B}^{\prime}}(G / P)$ is open and continuous (Mosak [23, Theorem 5.8]). Now $X(G / P)=(G / P)^{\wedge}$ is connected by abelian duality theory, since $G / P=\mathrm{R}^{n} \oplus A$ is an abelian torsion free group. Thus $\alpha \cdot X(G / P)$ is connected, for each $\alpha \in X(G)$, being the continuous 
image of $X_{(G / P)}$ under the map $\lambda \rightarrow a \cdot \lambda$. Now $\mathcal{U}_{a, P}$ equals $\alpha \cdot X_{(G / P)}$ by a result of Kaniuth and Schlichting [14, Lemma 4$]$, and $U_{\beta, P}^{\mathbb{B}}$ is the continuous image of $\mathcal{U}_{\alpha, P}$ under the canonical surjection $X_{(G / C)} \rightarrow X^{B}(G / C)$; $\alpha \rightarrow \beta=\int_{B^{-}} \alpha \iota d l$. Hence each $\mathcal{U}_{\beta, P}^{\mathscr{B}}$ is connected. Let $\mathcal{C}_{\beta}$ denote the connected component of $\beta$ in $\mathfrak{X}^{B}(G / C) \approx \mathfrak{X}^{B}(G)$. Then we have from the above that $\mathcal{C}_{\beta} \supset \mathrm{U}_{\beta, P}^{\mathbb{B}}$.

If $K$ is any compact $B$ invariant subgroup of $G$ containing $C$, then $K \subset P$ and $\mathrm{U}_{\beta, K}^{\mathfrak{B}}=\left\{\gamma \in \mathfrak{X}^{\mathfrak{B}}(G): \beta_{K}=\gamma_{K}\right\}$ is an open and closed subspace of $\mathcal{X}^{\mathscr{B}}(G)$ by Lemma $(2.2)$, and clearly $\mathcal{U}_{\beta, K}^{\mathbb{B}} \supset \mathcal{U}_{\beta, P}^{\mathbb{B}}$. Let $H=G / C$. The periodic subgroup $P(H)$ of $H$ equals $P / C$ and by [11, Theorem 3.16(2)], $P(H)$ is the union of all compact $B^{\prime}$ invariant subgroups of $H$. Taking pre-images under the canonical map $G \rightarrow G / C$, we see that $P$ is the union of all compact $B$ invariant subgroups $K$ of $G$ containing $C$. Hence we have $\bigcap_{K \supset C} U_{\beta, K}^{\mathbb{R}}=$ $U_{\beta, P}^{\mathbb{B}}$. Also the connected component $\mathcal{C}_{\beta} \subset \mathcal{U}_{\beta, K}^{\mathbb{B}}$ since $\mathcal{U}_{\beta, K}^{\mathbb{B}}$ is open and closed and has the character $\beta$ in common with $\mathcal{C}_{\beta}$. Thus $\mathcal{C}_{\beta} C$ $\bigcap_{K \supset C} \mathcal{U}_{\beta, K}^{\mathbb{B}}=\mathcal{U}_{\beta, P}^{\mathbb{B}}$, and as $\mathcal{U}_{\beta, P}^{\mathbb{B}}$ is connected, it follows that $\mathcal{C}_{\beta}=$ $\mathcal{U}_{\beta, P}^{\mathscr{B}}$. We have therefore proved

(2.3). Proposition. Suppose $G$ is an $[F C]_{\mathbb{B}}^{-}$group, where $\mathcal{B} \supset \mathscr{Y}(G)$. Then the connected component of $\beta \in \mathfrak{X}^{\mathbb{B}}(G)$ is $\mathcal{U}_{\beta, P}^{\mathbb{B}}$, where $P$ denotes the periodic subgroup of $G$.

(2.4). Corollary. Let $G$ be an $[F C]^{-}$group. Then the connected com-

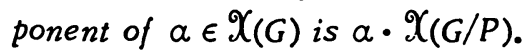

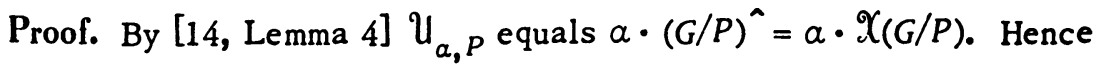
the result follows from Proposition (2.3).

As an application of (2.4) we prove the following result which is a slight generalization of part of Satz 3 in [13].

(2.5). Corollary. If $G$ is an $[F C]^{-}$group then $\mathfrak{X}(G)$ is discrete if and only if $G$ is compact.

Proof. If $\mathfrak{X}(G)$ is discrete it follows from (2.4) that the connected component $(G / P)^{\wedge}$ is trivial, so $G=P$ is periodic. By the structure theorem we have an exact sequence of topological groups $(e) \rightarrow K \rightarrow G \rightarrow D \rightarrow$ (e) where $D$ is a discrete periodic [FC] group, and $K$ is compact [18]. Since $X_{(D)}$ is naturally embedded in $X_{(G)}, X_{(D)}$ is discrete. Moreover, 
$D \in[F C] \cap[S I N]$, so that Satz 3 in [13] yields that $D$ is compact. Hence $G$ is compact. Conversely, if $G$ is compact it is well known that $\hat{G} \approx \Upsilon(G)$ is discrete [5].

Noting that, for $G \in[F C]^{-} \cap[S I N], X_{(G)}$ is compact iff $G$ is discrete [13, Satz 3], we derive as a consequence the following result, which is not true in general for locally compact groups, not even for second countable groups, as is shown in [3, Theorem (4.7)]. We have not succeeded in proving the result for $[F C]^{-}$groups. For $[F I A]^{-}$groups it follows immediately from the work of Mosak [23].

(2.6). Proposition. Let $G$ be an $[F I A]^{-}$group. If $\hat{G}$ is compact then $G$ is discrete.

Proof. The map $\phi \rightarrow \phi^{\#}, \hat{G} \rightarrow \mathfrak{X}(G)$, is a continuous surjection (Mosak [23]). Thus $X(G)$ is compact. Hence $G$ is discrete by [13, Satz 3$]$.

The next result was conjectured to hold for arbitrary locally compact groups. Then L. Baggett proved its validity for second countable groups [3], and recently A. I. Štern announced the result in complete generality [27].

We present a proof for $[F C]^{-}$groups because it is closely related to the material above.

(2.7). Proposition. Let $G$ be an $[F C]^{-}$group (not necessarily separable). Then $\hat{G}$ is discrete if and only if $G$ is compact.

Proof. If $\hat{G}$ is discrete, let, as before, $C$ be the intersection of all invariant neighborhoods of $e$ in $G$. Then $\hat{H}=(G / C)^{\wedge}$ is discrete. Since $H=$ $G / C$ is an $[F I A]^{-}$group the map $t: \hat{H} \rightarrow X(H)$ given by $\phi \rightarrow \phi^{\#}$, where $\phi^{\#}(b) I=\int_{g(H)^{-}} \phi \iota(h) d \iota$ is open and continuous onto $X(H)$. Hence $X(H)$ is discrete, so that $H$ is compact. Since $C$ is compact it follows that $G$ is compact.

The converse assertion ( $G$ compact implies $\hat{G}$ discrete) is well known [5].

It was proved in [10] that [Z] groups are first countable if and only if their character spaces $X(G)$ are $\sigma$-compact. We will now prove the "only if part" of this result for $[F D]^{-}$groups, whereas the "if part" only can hold for $[F I A]^{-}$groups in view of (2.1).

(2.8). Proposition. Suppose $G$ is an $[F D]^{-}$group. If the character space $X(G)$ is o-compact then the $[F I A]^{-}$quotient group $G / C$ is first count- 
able (where $C$ denotes the intersection of all closed invariant neighborhoods of $e$ ). Conversely, if $G \in[F D]^{-}$and $X(G)$ is $\sigma$-compact then the group $G$ is. first countable.

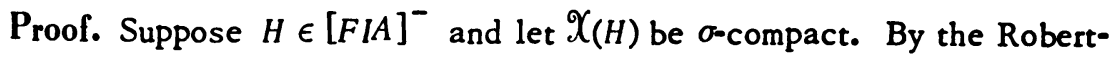
son structure theorem $H$ satisfies an exact sequence of topological groups $(e) \rightarrow K \rightarrow H \rightarrow R^{n} \oplus D \rightarrow(e)$ where $D$ is a discrete [FC] group and $K$ is compact and normal in $H$.

Now the continuous restriction map $a \rightarrow \alpha_{K}, X(H) \rightarrow X^{H}(K)$ is surjective (Kaniuth [13, Satz 4]) so that $\Upsilon^{H}(K)$ is $\sigma$-compact. ${ }^{(9)}$ Also, $\mathfrak{X}^{H}(K)$ is discrete since $K$ is compact, hence $\mathscr{X}^{H}(K)$ is countable. Moreover, each $\beta \in \mathfrak{X}^{H}(K)$ is of the form $\beta=1 / n \Sigma_{\tau \in \theta^{\tau}}$ where $\theta$ is some $H$-orbit in $X_{(K)}$ and $n$ denotes its finite order (see e.g. the proof of Lemma (2.2)). Thus $X(K) \approx \hat{K}$ is countable and hence $K$ is first countable. Therefore $K$ and $H / K=\mathbf{R}^{n} \oplus D$ are first countable and this implies that $H$ itself is first countable (see e.g. $[10$, Lemma (2.1)]). Now the first part of the proposition follows by letting $H=G / C$.

Conversely, if $G \in[F D]^{-}$is first countable and $G^{\prime}$ denotes its commutator subgroup, let $K$ be any compact open subgroup of the periodic subgroup $P$ of $G$ and assume that $K$ is $G$-invariant [18]. Since $P \supset \bar{G}^{\prime}$ and $\bar{G}^{\prime}$ is compact we may also assume that $K \supset \bar{G}^{\prime}$. By hypothesis $K$ is first countable, hence $X(K) \approx \hat{K}$ is countable. If $\alpha \in X(G)$, we have $a_{K}=1 / m \Sigma_{\theta^{\tau}}$ where $\theta$ is some $G$-orbit in $\Upsilon(K)$ and $m$ is the finite order of $\theta$ (see the proof of Lemma (2.2)). Since there are only countably many orbits, there are only countably many restrictions $a_{K}$. Thus $X_{(G)}$ is the disjoint union of countably many $\mathcal{U}_{a, K}$, and each $\mathcal{U}_{a, K}$ is of the form $\alpha \cdot(G / K)^{\wedge}$ since $G / K$ is abelian [14, Lemma 4]. By abelian duality theory $(G / K)^{\wedge}$ is $\sigma$-compact ( $G / K$ is first countable by the hypothesis). Hence it is clear that each $a \cdot(G / K)^{\wedge}$ is $\sigma$-compact, being the continuous image of $(G / K)^{\wedge}$ under the map $\lambda \rightarrow \alpha \cdot \lambda$, thus $X(G)$ is the countable union of $\sigma$-compact spaces $\alpha \cdot(G / K)^{\wedge}$, and it follows that $\mathcal{X}(G)$ is itself $\sigma$-compact.

In order to establish our next result we shall need

(2.9). Lemma [14, Lemma 6]. Let $G$ be an [FC] group, and suppose the periodic subgroup $P$ is open in $G$. Then the compact-open subsets $A$ of $X(G)$ are exactly the sets of the form $A=\bigcup_{i=1}^{r} \mathcal{U}_{a_{i}, N}$, where $N$ is some

(9) We let $x^{H}(K)=x^{g(H)} K_{(K)}$. 
compact, open, and normal subgroup of $G$.

(2.10). Proposition. Let $G$ be an $[F I A]^{-}$group. Then the character space $X(G)$ has finitely many connected components if and only if the periodic subgroup $P$ of $G$ is finite.

Proof. Clearly, if $P$ is finite there are only a finite number of $G$-orbits in $X(P) \approx \hat{P}$. Hence there are finitely many $\mathcal{C}_{a}=\alpha \cdot(G / P)^{\wedge}$ since different connected components $\mathcal{C}_{\alpha}$ correspond to different orbits.

Conversely, suppose $\mathfrak{X}(G)$ has finitely many connected components, and let $K$ be any compact open subgroup of $P$ and characteristic in $G$ [18]. There are only a finite number of different sets $\mathcal{U}_{a, K}=\left\{\beta \in X_{(G):} a_{K}=\beta_{K}\right\}$, since each $\mathcal{U}_{a, K} \supset \mathcal{C}_{\alpha}$, and since we have $\mathcal{C}_{\alpha}=\mathcal{C}_{\beta}$ or $\mathcal{C}_{\alpha} \cap \mathcal{C}_{\beta}=\varnothing$, for $\alpha, \beta \in X(G)$. We shall see that to each $G$-orbit in $X_{(K)}$ there corresponds a

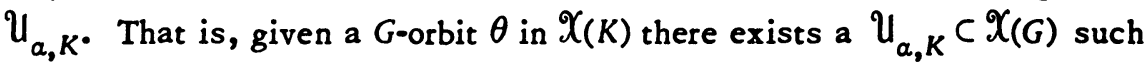
that each character in $\mathcal{U}_{\alpha, K}$ restricts to $K$ as a sum $1 / n \Sigma \beta$ taken over $\theta$. As soon as this is proved, it follows that $X(K)$ has finitely many orbits. Moreover, for fixed $\gamma \in X(K)$, the map $\mathscr{I}(G)^{-} \rightarrow X(K) ; \iota \rightarrow \gamma \iota$ is continuous $[23,5.6]$, and since $\mathscr{Y}(G)^{-}$is compact and $X(K)$ discrete, it follows that the G-orbits in $X(K)$ are finite. Thus it follows that $X(K) \approx \hat{K}$ is finite. By a result of $\mathrm{L}$. Baggett [2] we conclude that $K$ is finite.

We will now prove the statement above. Let $\theta$ be a $G$-orbit in $X_{(K)}$, and

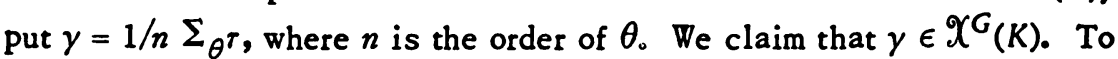
see this, suppose $\lambda \gg 0$ and $G$-invariant on $K$, and that $\lambda \ll \gamma$. We have $\lambda=\Sigma_{\sigma \in \mathscr{X}(K)} m(\sigma) \sigma$, and we shall write $\lambda=\lambda_{\theta}+\lambda_{\theta}^{\perp}$, where $\lambda_{\theta}$ is the part of $\lambda$ concentrated on the orbit $\theta$. Suppose ad absurdum that $\lambda_{\theta}^{\perp} \neq 0$. Since $\lambda_{\theta}^{\perp}$ is positive definite and square-integrable, we have $\lambda_{\theta}^{\perp}=f^{*} * f$, for some continuous (positive definite) function $f$ on $K,[5,13.8 .6]$. Hence, by the orthogonality relations,

$$
\begin{aligned}
0 & \leq \int_{K}\left(f^{*} * f\right)(k)(y-\lambda)^{-}(k) d k \\
& =\int_{K} \lambda_{\theta}^{\perp}(k)\left(\gamma(k)^{-}-\lambda(k)^{-}\right) d k=\int_{K}-\left|\lambda_{\theta}^{\perp}(k)\right|^{2} d k<0 .
\end{aligned}
$$

Hence $\lambda=\lambda_{\theta}=\Sigma_{\theta} m(\sigma) \sigma$. Now $x \cdot \lambda(k)=\lambda(k), x \in G, k \in K$, so that $m(x \cdot \sigma)=m(\sigma)$ by uniqueness of the basis expansion. Thus $\lambda=$ $\lambda(e)\left(1 / n \Sigma_{\theta} \sigma\right)=\lambda(e) \gamma$, and we have proved that $\gamma$ is an extreme point, i.e. $\gamma \in \mathfrak{X}^{G}(K)$ 。 By Kaniuth [13, Satz 4], there exists an $\alpha \in \mathfrak{X}(G)$ such that the restriction $\alpha_{K}$ equals $\gamma$. Hence the orbit $\theta$ corresponds to $\mathcal{U}_{a, K}$, and we 
have seen that this implies that $K$ is finite. By assumption, $K$ was open in $P$, so $P$ is discrete. By Liukkonen [18, Proposition 2.4] $G=\mathbf{R}^{n} \oplus H$, where $H$ is a discrete $[F C]$ group. Also, $X_{(H)}$ has finitely many connected components, and therefore each of them is open. In particular $(H / P)^{\wedge}$ is open and compact, and Lemma (2.9) yields that $(H / P)^{\wedge}=U_{1}^{r} \mathcal{U}_{a_{i}, N}$ where $N$ is compact and normal in $H$. Since $(H / P)^{\wedge}$ is connected $r=1$ and $(H / P)^{\wedge}=$ $X(H / N)$, which implies $H / N$ is aperiodic (2.12). Hence $P=N$ and we have that $P$ is finite.

(2.11). Corollary. Let $G$ be an $[F C]^{-}$group with periodic subgroup $P$. Let $C$ denote the intersection of all invariant neighborboods of $e$ in $G$. Then $X(G)$ bas finitely many connected components if and only if $C$ bas finite index in $P$.

Proof. This follows immediately from (2.1) and (2.10).

(2.12). Proposition. Let $G$ be an $[F C]^{-}$group, and let $C$ be the intersection of all invariant neighborhoods of $e$. Then $G / C$ is aperiodic if and only if $X_{(G)}$ is connected.

Proof. Since aperiodic $[F C]^{-}$groups are of the form $\mathbf{R}^{n} \oplus A$ where $A$ is discrete, abelian and torsion free, the only if part follows from the abelian theory and the fact that $X_{(G)} \approx X(G / C)$.

Conversely, suppose $\mathscr{X}(G)$ is connected. By (2.4) we then have $\mathscr{X}(G) \approx$ $X(G / C)=(G / P)^{2}$, and since $G / C$ is a $[S I N]$ group the characters in $X(G / C)$ separate the points of $G / C$ (Dixmier $[5,17.3 .6]$ ). Thus $P / C$ is trivial, and $G / C=G / P$ is aperiodic.

Connectedness being the farthest, topologically speaking, from totally disconnectedness, one can ask whether periodic groups are the ones possessing totally disconnected character space.

(2.13). Proposition. Let $G$ be an $[F C]^{-}$group. Then $X(G)$ is totally disconnected if and only if $G$ is periodic.

Proof. If $G=P$ then clearly the character space $X_{(G)}$ is totally disconnected (2.4).

Conversely, if $X_{(G)}$ is totally disconnected, $(G / P)^{\wedge}$ is trivial, so $G / P=$ $(e)$ by the Gel'fand-Raikov theorem. We close this section with an example.

(2.14). Example. Let $G$ be the semidirect product of the integers $\mathbf{Z}$ and the compact group $\mathbf{T}^{\infty}=\Pi_{-\infty}^{+\infty} \mathbf{T}_{k}$ where $\mathbf{T}_{k}=\mathbf{R} / \mathbf{Z}$ for each $k \in \mathbf{Z}$, and where $Z$ acts on $T^{\infty}$ by shifting the coordinates: $\alpha: Z \rightarrow \operatorname{Aut}\left(\mathbf{T}^{\infty}\right), \alpha(n)\left(t_{k}\right)_{k}=$ 
$\left(t_{k+n}\right)_{k}$. Then $\hat{G}$ can be computed by Mackey's theory. If $\omega \in\left(\mathrm{T}^{\infty}\right)^{\wedge}$ is nontrivial, the stability group $K_{\omega}$, under the action of $G$ on $\left(T^{\infty}\right)^{\wedge}$ by inner automorphisms, equals $\mathrm{T}^{\infty}$. Hence $\hat{G}$ consists of the induced infinite dimensional representations $\omega^{G}$ and $\hat{G}_{0}=\left\{\pi \in \hat{G}: \pi_{T^{\infty}}\right.$ is trivial $\} \approx \mathrm{Z}^{\wedge}$. Therefore the space of finite dimensional irreducible representations, $\hat{G}_{0}$, forms a connected subspace of $\hat{G}$. We note that $G$ is an $[F C]^{-}$group by the structure theorem of Robertson. Also, $G$ is type $I$. This follows from the Mackey theory since the stability groups equal $\mathrm{T}^{\infty}$ (except for $\omega=1$ ) and since $\mathbf{Z}$ and $\mathrm{T}^{\infty}$ both are type $\mathrm{I}$, being abelian [21, Theorem 8.4].

Since separable $[F I A]^{-}$groups of type I have only finite dimensional irreducible representations, $G$ is not a [SIN] group [23]. Actually, as is easily seen, the intersection of all invariant neighborhoods of $e$ in $G$ equals $\mathrm{T}^{\infty}$. Hence we have $\mathscr{X}(G)=\hat{G}_{0}$ so that $G$ is an $[F C]^{-}$group where the periodic subgroup is infinite $\left(P=\mathrm{T}^{\infty}\right)$ but where $X(G)$ is connected; compare with Proposition (2.10).

Observe also that $\mathcal{X}(G)$ is compact without $G$ being discrete; see Proposition (2.6).

3. Further results on $\mathfrak{X}^{\mathbb{B}}(G)$. We continue our extension of results from the duality theory of abelian groups and $[Z]$ groups. Before we turn to characterizations of $\sigma$-compact and compactly generated $[F C]^{-}$groups, we shall need the following useful lemma.

(3.1). Lemma. Let $G$ be an $[F C]^{-}$group, and let $N$ be a compact normal subgroup of $G$ such that the quotient $G / N$ is abelian and first countable. Let $\beta \in \mathfrak{X}(G)$. Then the map

$$
\psi: X(G / N) \rightarrow \beta \cdot X(G / N), \quad \lambda \rightarrow \beta \cdot \lambda,
$$

is an open and continuous surjection.

Proof. The map $\psi$ is clearly continuous (in the topology of uniform convergence on compacta in $G / N$ ) and surjective.

To prove that $\psi$ is open, we first observe that $\mathcal{X}(G / N)=(G / N)^{\wedge}$ is the character group of the abelian and first countable group $G / N$; hence $(G / N)$ ^ is $\sigma$-compact. Next, we give $\beta \cdot X(G / N)$ the structure of a group by letting

$$
\left(\beta \cdot \lambda_{1}\right) *\left(\beta \cdot \lambda_{2}\right)=\beta \cdot\left(\lambda_{1} \lambda_{2}\right), \text { and }\left(\beta \cdot \lambda_{1}\right)^{-1}=\beta \cdot \lambda_{1}^{-1} ;
$$

$\lambda_{1}, \lambda_{2} \in X(G / N)$. It is easy to verify that the map

$$
\left(\beta \cdot \lambda_{1}, \beta \cdot \lambda_{2}\right) \rightarrow \beta \cdot\left(\lambda_{1} \lambda_{2}^{-1}\right) ; \beta \cdot X(G / N) \times \beta \cdot X(G / N) \rightarrow \beta \cdot \chi(G / N)
$$


is continuous in the topology of uniform convergence on compacta. Hence $\beta \cdot \Upsilon(G / N)$ becomes a topological group with the above multiplication.

Moreover,

and

$$
\psi\left(\lambda_{1} \lambda_{2}\right)=\beta \cdot\left(\lambda_{1} \lambda_{2}\right)=\left(\beta \cdot \lambda_{1}\right) *\left(\beta \cdot \lambda_{2}\right)=\psi\left(\lambda_{1}\right) * \psi\left(\lambda_{2}\right)
$$

$$
\psi\left(\lambda_{1}\right)^{-1}=\left(\beta \cdot \lambda_{1}\right)^{-1}=\psi\left(\lambda_{1}^{-1}\right) ; \quad \lambda_{1}, \lambda_{2} \in X(G / N),
$$

so that $\psi$ is a continuous homomorphism of the $\sigma$-compact locally compact group $X(G / N)$ onto the topological group $\beta \cdot \mathscr{X}(G / N)$. By Kaniuth [13, Satz 1], $\mathcal{X}(G)$ is a locally compact Hausdorff space, and by Lemma (2.2) $\beta \cdot X(G / N)$ is an open and closed subspace of $\mathscr{X}(G)$. Hence $\beta \cdot X(G / N)$ is itself a locally compact Hausdorff space. We may now apply Theorem 5.29 of Hewitt and Ross [12] to conclude that $\psi$ is an open map.

(3.2). Proposition. Let $G$ be an $[F D]^{-}$group such that $G / \bar{G}^{\prime}$ is first countable (where $G^{\prime}$ denotes the commutator subgroup of $G$ ). Then $G$ is $\sigma$ compact if and only if $X_{(G)}$ is first countable.

Proof. If $X_{(G)}$ is first countable, so is $\left(G / \bar{G}^{\prime}\right)^{\wedge}$ since it is a subspace of $\Upsilon(G)$. Hence $G / \bar{G}^{\prime}$ is $\sigma$-compact by the abelian theory. Since by hypothesis $\bar{G}^{\prime}$ is compact, $G$ is $\sigma$-compact.

Conversely, suppose $G$ is $\sigma$-compact. Then clearly $G / \bar{G}^{\prime}$ is $\sigma$-compact, and hence its character group $\left(G / \bar{G}^{\prime}\right)^{\wedge}=\mathfrak{X}\left(G / \bar{G}^{\prime}\right)$ is first countable. By Lemma (3.1) $a \cdot X\left(G / \bar{G}^{\prime}\right)$ is first countable for every $a \in X(G)$. Since each $a \cdot X\left(G / \bar{G}^{\prime}\right)$ is open in $X(G)$, it follows that $X(G)$ is first countable.

A topological space is said to be locally Euclidean if each of its points is contained in a neighborhood which is homeomorphic with $\mathbf{R}^{n}$ for some $n$.

(3.3). Proposition. Let $G$ be an $[F C]^{-}$group. Then the character space $X(G)$ is locally Euclidean if $G$ is compactly generated. If $G$ is an [FD]group and the character space $X(G)$ is locally Euclidean then $G$ is compactly generated.

Proof. Suppose $G$ is compactly generated. Then $G$ satisfies an exact sequence of topological groups $(e) \rightarrow K \rightarrow G \rightarrow \mathbf{R}^{n} \oplus A \rightarrow(e)$ where $K$ is the periodic subgroup which contains the closed commutator $\bar{G}^{\prime}$, is compact, and $A$ is discrete, abelian, finitely generated, and torsion free [11, Theorem (3.20)]. By abelian duality theory $(G / K)^{\wedge}=\mathfrak{X}(G / K)$ is locally Euclidean, and the proof of (3.1) shows that the connected components $\alpha \cdot X(G / K)$ are locally compact groups. By (3.1) the map $\psi: \lambda \rightarrow a \cdot \lambda$ is an open and 
continuous group homomorphism of $X(G / K)$ onto $a \cdot X(G / K)$. Hence the topological groups $\mathscr{X}(G / K) / \operatorname{ker} \psi$ and $\alpha \cdot \mathfrak{X}(G / K)$ are isomorphic. Since the first is a Lie group, so is $\alpha \cdot \mathscr{X}(G / K)$. Now each connected component is open in $X_{(G)}$ since $K$ is compact (2.2) and it follows that $X_{(G)}$ is locally Euclidean.

Conversely, suppose $X(G)$ is locally Euclidean and $G \in[F D]^{-}$. Then $\left(G / \bar{G}^{\prime}\right)^{\wedge}$ is open in $X(G)$ and hence it is locally Euclidean. Thus $G / \bar{G}^{\prime}$ is compactly generated by the abelian theory. Since $\bar{G}^{\prime}$ is compact, $G$ must be compactly generated.

Local connectedness and property $(L)$. Let $G$ be a locally compact group and let $\mathscr{B}$ be a subgroup of $\mathscr{Q}(G)$ containing the group $\mathscr{G}(G)$ of inner automorphisms. $G$ is said to have property $(L)_{\mathscr{B}}$, or to be an $(L)_{\mathbb{B}}$ group, if each compact subset $M$ of $G$ is contained in an open, compactly generated, B-invariant subgroup $H$ of $G$ such that the quotient $G / H$ is torsion free. If $B=\mathscr{Y}(G)$ and $G$ is abelian this definition reduces to the usual one (Pontrjagin [24]). In that case we shall use the notation ( $L$ ) group.

Our first task is to prove that for $[F C]_{\mathbb{B}}^{-}$groups $G$ possessing property $(L)_{\mathscr{B}}$ the $B$ character space $\mathscr{X}^{\mathscr{B}}(G)$ is locally connected. In the abelian case this result (and its converse) was proved by K. Fan [6]. Although his proof can be generalized to $[F C]_{\mathscr{B}}^{-}$groups, we will use a different approach (giving a shorter proof), which utilizes our results from $\$ \S 2$ and 3 concerning the structure of $X^{\mathbb{B}}(G)$, and Fan's result. Before going on, we note that the following result was proved for [Z] groups in [10].

(3.4). Proposition. Let $G$ be an $[F C]_{\bar{B}}^{-}$group (such that the quotient group $G / P$ is first countable, where $P$ denotes the periodic subgroup of $G) .(10)$ Then $X^{B}(G)$ is locally connected if $G$ possesses property $(L)_{B}$.

Proof. Let $U$ be any compact subset of the periodic subgroup $P$ of $G$. Since $G$ is an $(L)_{B}$ group, $U$ is contained in a compactly generated, open, B-invariant subgroup $H$ of $G$ with torsion free quotient $G / H$. Clearly, $P \subset H$; in fact, any $x \in P \backslash H$ would generate a compact subgroup of $P$ with nontrivial compact image in $G / H$ under the canonical map $G \rightarrow G / H$, contradicting the fact that $G / H$ is torsion free (aperiodic). It is also clear that $P$ is the periodic subgroup of the $[F C]^{-}$group $H$, and since $H$ is compactly generated $P$ must be compact [11, Theorem 3.20].

Let now $C_{B}$ be the intersection of all $B$-invariant neighborhoods of $e$. Recall that the canonical map $\psi$ of $\mathscr{X}\left(G / C_{\mathscr{B}}\right)$ onto $X^{B^{\prime}}\left(G / C_{B}\right)$ is open and

(10) $G / P=\mathbf{R}^{n} \times D$ where $D$ is discrete, is always first countable. 
continuous (Mosak $[23,5.8]$ ). Put $M=G / C_{g}$ and let $P(M)$ be the periodic subgroup of $M$. If $\alpha \cdot \lambda \in \alpha \cdot(M / P(M)), \alpha \in X(M)$ and $\lambda \in(M / P(M))$, then

$$
[\psi(\alpha \cdot \lambda)](p)=\int_{\overline{\mathscr{D}}^{\prime}} \alpha \circ \iota(p) \lambda \circ \iota(p) d \iota=\int_{\overline{\mathscr{B}}^{\prime}} \alpha \circ \iota(p) d \iota=(\psi \alpha)(p),
$$

all $p \in P(M)$, since $\iota(p) \in P(M)$ whenever $\iota \in B^{\prime}$, and since the restriction $\lambda_{P(M)} \equiv 1$. This implies that $\psi$ maps $\alpha \cdot(M / P(M))^{\wedge}=\mathcal{U}_{\alpha, P(M)}$ onto $\mathcal{U}_{\psi a, P(M)}^{B^{\prime}}$ in $X^{\prime}{ }^{\prime}(M)$. Since $P(M)$ is compact, each $\alpha \cdot(M / P(M))$, $\alpha \in X(M)$, is open in $X_{(M)}$ (Lemma (2.2)) and it suffices to prove that all the connected components $\alpha \cdot(M / P(M))^{\wedge}$ of $X(M)$ are locally connected in order to show that $X^{B^{\prime}}(M) \approx X^{\mathscr{B}}(G)$ is locally connected.(11)

The facts that $M / P(M)$ is abelian, $P(M)$ is compact $\left(P(M)=P / C_{B}\right.$ and $P$ is compact as shown above), and $M / P(M)$ is first countable make Lemma (3.1) available. Hence the map $\lambda \mapsto \alpha \cdot \lambda$ of $(M / P(M))^{\wedge}$ onto $\alpha \cdot(M / P(M))^{\wedge}$ is open, and it is sufficient to prove that $(M / P(M))^{\wedge}$ is locally connected. Noting that $M / P(M)$ is of the form $\mathbf{R}^{k} \oplus A$ where $A$ is discrete, abelian, and aperiodic, the problem is reduced to showing that $\mathbf{R}^{k} \oplus A$ is an $(L)$ group by the result of $\mathrm{K}$. Fan for abelian groups.

Let therefore $F$ be a compact subset of $\mathbf{R}^{k} \oplus A$, and let $\pi: M \rightarrow$ $M / P(M)=\mathbf{R}^{k} \oplus A$ be the canonical map. Then $\pi^{-1}(F)$ is compact and hence is by hypothesis contained in a compactly generated open normal subgroup $K$ of $M$ such that the quotient $M / K$ is torsion free. Hence $\pi(K)$ is a compactly generated subgroup of $\mathbf{R}^{k} \oplus A$ and, moreover, $K$ contains $P(M)$. Thus

$$
\left(\mathbf{R}^{k} \oplus A\right) / \pi(K) \approx(M / P(M)) /(K / P(M)) \approx M / K,
$$

and it follows that $\left(\mathrm{R}^{k} \oplus A\right) / \pi(K)$ is torsion free. Since $\pi(K)$ clearly contains $F$, we have seen that $\mathbf{R}^{k} \oplus A$ possesses property $(L)$, and it follows that $\left(\mathbf{R}^{k} \oplus A\right)^{\wedge}=(M / P(M))^{\wedge}$ is locally connected.

We turn now to the converse of Proposition (3.4), assuming $B=\mathscr{G}(G)$ and $G \in[F D]^{-}$. If $X(G)$ is locally connected, $\mathcal{C}_{\iota}=(G / P)^{\wedge}$ contains an (open) connected neighborhood of the identity character $\iota$, so $(G / P)^{\wedge}$ is open in $X(G)$. As $P$ contains the commutator $G^{\prime},(G / P)^{\wedge}$ is open in $\left(G / \bar{G}^{\prime}\right)^{\wedge}$. Also $(G / P)^{\wedge} \approx\left(G / \bar{G}^{\prime} / P / \bar{G}^{\prime}\right)^{\wedge}$, so $P / \bar{G}^{\prime}$ is compact by abelian theory, and it follows that $P$ is compact.

Suppose in addition $G$ is an $[S I N]$ group. Then $G$ satisfies an extension of topological groups $(e) \rightarrow \mathbf{R}^{n} \oplus P \rightarrow G \stackrel{\pi}{\rightarrow} A \rightarrow(e)$ where $A$ is discrete

(11) The author is indebted to R. Mosak for pointing out a gap in an earlier version of the above argument. 
abelian and torsion free [18, Theorem 2.13]. Let $M$ be a compact subset of $G$. Since $A$ is discrete, $\pi(M)$ must be finite. Let now $F$ be the set of those elements $a$ in $A$ for which there is a positive integer $n$ such that $a^{n}$ is in the subgroup generated by $\pi(M)$. Hence $F$ is a discrete torsion free group of finite rank. Now $\hat{F}$ can be identified with the quotient group $\hat{A} /(A / F)^{\wedge}$ and $A$ is locally connected, hence $\hat{F}$ is locally connected. By a lemma of Pontrjagin $[24, \$ 38], F$ must be finitely generated.

Let $H=\pi^{-1}(F)$. Clearly $H$ is open in $G$, since $A$ is discrete. As $F$ is finitely generated and $\mathbf{R}^{n} \oplus P=\operatorname{ker} \pi$ is compactly generated, $H$ is compactly generated. Moreover, $A / F$ is torsion free from the definition of $F$. Hence $G / H$ is torsion free, being isomorphic with $A / F$. Therefore the compact set $M$ is contained in the compactly generated open subgroup $H$ of $G$ with torsion free quotient $G / H$. Finally we note that $H$ is invariant in $G$.

Let $G$ be an arbitrary $[F D]^{-}$group and put $C=$ the intersection of all invariant neighborhoods of the identity $e$. Then $D=G / C$ is an [SIN] group and $X(G)$ is homeomorphic to $\Upsilon(D)$. Suppose $D$ is an $(L)$ group and let $M \subset G$ be compact. If $\omega: G \rightarrow G / C$ is the canonical epimorphism, $\omega(M)$ is compact in $G / C$ and hence contained in an open, compactly generated, invariant subgroup $H^{*}$ of $D$ with torsion free quotient $D / H^{*}$. Then $\omega^{-1}\left(H^{*}\right)=$ $H$ is an invariant subgroup of $G$ containing $M$, and possesses the other crucial properties: it is compactly generated, open in $G$ and the quotient $G / H$ is torsion free. We have proved

(3.5). Proposition. Let $G$ be an $[F D]^{-}$group and suppose $X(G)$ is locally connected. Then $G$ is an $(L)$ group.

\section{REFERENCES}

1. L. Auslander and C. C. Moore, Unitary representations of solvable Lie groups, Mem. Amer. Math. Soc. No. 62 (1966). MR 34 \#7723.

2. L. Baggett, A note on groups with finite dual spaces, Pacific J. Math. 31 (1969), 569-572. MR 41 \#3658.

3. - A separable group having a discrete dual space is compact, $\mathrm{J}$ 。 Functional Analysis 10 (1972), 131-148.

4. J. Dixmier, Les algèbres dans l'espace hilbertien (Algèbres de von Neumann), Gauthier-Villars, Paris, 1957. MR 20 \#1234.

5. - Les $C^{*}$-algèbres et leurs représentations, Cahiers Scientifiques, fasc. 29, Gauthier-Villars, Paris, 1964. MR 30 \#1404.

6. K. Fan, On local connectedness of locally compact abelian groups, Math. Ann. 187 (1970), 114-116. MR 41 \#7022.

7. J. M. G. Fell, A new proof that nilpotent groups are CCR, Proc. Amer. Math. Soc. 13 (1962), 93-99. MR 24 \#A3238.

8. - Weak containment and induced representations of groups, Canad. J. Math. 14 (1962), 237-268. MR 27 \#242.

9. F. Greenleaf and M. Moskowitz, Cyclic vectors for representations asso- 
ciated with positive definite measures: Nonseparable groups, Pacific J. Math. 45 (1973), 165-186.

10. S. Grosser, R. Mosak and M. Moskowitz, Duality and harmonic analysis on central topological groups. I, II, Indag. Math. 35 (1973), 66-77, 78-91.

11. S. Grosser and M. Moskowitz, Compactness conditions in topological groups, J. Reine Angew. Math. 246 (1971), 1-40. MR 44 \#1766.

12. E. Hewitt and K. A. Ross, Abstract harmonic analysis. Vol. I: Structure of topological groups. Integration theory, group representations, Die Grundlehren der math. Wissenschaften, Band 115, Academic Press, New York; Springer-Verlag, Berlin, 1963. MR 28 \#158.

13. E. Kaniuth, Zur harmonischen Analyse klassenkompakter Gruppen, Math. Z. 110 (1969), 297-305. MR 41 \#8591.

14. E. Kaniuth and G. Schlichting, Zur harmonischen Analyse klassenkompakter Gruppen. II, Invent. Math. 10 (1970), 332-345. MR 47 \#5521.

15. A, Kleppner and R. Lipsman, The Plancherel formula for group extensions, Ann. Sci. École Norm. Sup. 5 (1972), 459-516.

16. A. A. Kirillov, Unitary representations of nilpotent Lie groups, Uspehi Mat. Nauk 17 (1962), no. 4 (106), 57-110 = Russian Math. Surveys 17 (1962), no. 4, 53104. MR $25 \# 5396$.

17. H. Leptin, $Z$ ur harmonischen Analyse klassenkompakter Gruppen, Invent. Math. 5 (1968), 249-254. MR 38 \#2257.

18. J. Liukkonen, Dual spaces of locally compact groups with precompact conjugacy classes, Trans. Amer. Math. Soc. 180 (1973), 85-108.

19. J. Liukkonen and R. Mosak, Harmonic analysis and centers of group algebras, Trans. Amer. Math. Soc. 195 (1974), 147-163.

20. - The primitive dual space of $[\mathrm{FC}]^{-}$groups, J. Functional Analysis 15 (1974), 27?)-296.

21. G. W. Mackey, Unitary representations of group extensions. I, Acta. Math. 99 (1958), 265-311. MR 20 \#489.

22. C. Moore and J. Wolf, Square integrable representations of nilpotent groups, Trans. Amer. Math. Soc. 185 (1973), 445-462.

23. R. D. Mosak, The $L^{1}$ - and $C^{*}$-algebras of $[F I A]_{B}^{-}$groups, and their representations, Trans. Amer. Math. Soc. 163 (1972), 277-310. MR 45 \#2096.

24. L. S. Pontrjagin, Continuous groups, GITTL, Moscow, 1954; English transl., Gordon and Breach, New York, 1966. MR 17, 171; 34 \#1439.

25. L. C. Robertson, A note on the structure of Moore groups, Bull。Amer. Math. Soc. 75 (1969), 594-599. MR 39 \#7027.

26. I. Schochetman, Topology and the duals of certain locally compact groups, Trans. Amer. Math. Soc. 150 (1970), 477-489. MR 42 \#422.

27. A. I. Štern, Separable locally compact groups with discrete support for the regular representation, Dokl. Akad. Nauk SSSR 198 (1971), 1287-1290 = Soviet Math. Dokl. 12 (1971), 994-998. MR 44 \#6910.

SCHOOL OF MATHEMATICS, UNIVERSITY OF MINNESOTA, MINNEAPOLIS, MINNESOTA 55455 Norway

Current address: Matematisk Institutt, Universitetet i Oslo, Blindern, Oslo 3, 